\title{
DIFFUSION AND METAL ABUNDANCES IN HOT WHITE DWARFS
}

\author{
Gérard Vauclair \\ Observatoire Midi-Pyrénées \\ 14 av. E. Belin, 31400 TOULOUSE, France
}

\section{Introduction}

While the efficiency of gravitational settling to produce chemically pure atmospheres in white dwarf stars was outlined for the first time 30 years ago (Schatzman 1958), the competing role of the radiation flux in the hot white dwarfs was considered only 10 years ago (Fontaine and Michaud 1979; Vauclair, Vauclair and Greenstein 1979). At that time, there was more motivation to understand how metals could reappear in the long lived cool non DA white dwarfs, where diffusion time scales are shorter by orders of magnitude than evolutionary time scales. Various processes were invoked to help restore some metal content in the white dwarf atmospheres: convection mixing and dredge up, accretion of interstellar matter. In cool white dwarfs, the radiative acceleration is negligeable in the diffusion process; this is not the case at the hot end of the sequence where radiation may balance gravity. The short lived hot white dwarfs just started to become exciting with the contemporary discoveries that i) some show metallic lines in their spectra, both hydrogen rich and hydrogen poor; ii) some of these are pulsating. In the following years, the number of hot white dwarfs revealing trace abundance of metals has increased, mainly owing to IUE observations.

I do not intend here to make an exhaustive presentation of the observations. Such discussions may be found in recent reviews (Vauclair and Liebert 1987; Shipman 1987) and are also presented at this colloquium. In this paper, I prefer to stay on the theoretical side and present the results of the diffusion theory as they have been obtained, either to predict metal abundances to be expected in hot white dwarfs or to interpret observations of particular stars. Of course, the discovery of metallic lines in high dispersion IUE spectra of hot white dwarfs, as predicted by theory, was somewhat encouraging. However, now that so many hot white dwarfs show metallic lines, the theory has to be refined. Proper comparison of the observations with the theory should tell us about important mechanisms acting at this evolutionary phase. Important questions are still to be solved: i) what are the various channels conducting a dying star to the white dwarf final stage of evolution? ii) what is the link between the presence of metals in the atmosphere of the PG1159 stars and their instability? iii) what is the role of mass loss, selective or not, in that part of the HR diagram? The chemical composition observed in the atmosphere is affected by both the prior 
evolution of the stars and the presently acting physical mechanisms. A detailed description of the physical mechanisms in action at this evolutionary phase should lead to a better understanding of the questions quoted above. IUE has opened a new and rich area of research but is limited in size. With the Hubble Space Telescope we hope to get of a statistically significant sample of hot white dwarf high resolution spectra. This is one of the strong motivations to pursue detailed diffusion computations.

In the presence of pressure, temperature, concentration gradients and radiation flux, an ion of a trace element is submitted to forces induced by the various gradients, and by the absorption of selective parts of the radiation flux through lines. It is well known (see for instance Vauclair 1983) that as long as the abundance of the diffusing particles is small, i.e. that the opacity due to these particles is much smaller than the total opacity of the gas, the radiative acceleration on the diffusing particles does not depend on their abundance. In the reverse case, when the absorption of radiation due to the diffusing particles cannot be neglected compared to the total opacity, the radiative acceleration is affected by saturation and becomes dependent upon the abundance. It is for this reason that equilibrium abundances may be reached. A large abundance of a given element will not be generally supported by the radiation flux as there are too many ions willing to absorb a limited number of photons at the ions absorption line wavelengths. As a consequence the upward radiative acceleration is not able to compensate the downward acceleration due to pressure and temperature gradients. The ions diffuse downwards. Doing so, their abundance decreases, their absorption lines progressively desaturate and the radiative acceleration increases. When the radiative acceleration may just balance the downward acceleration, the ions reach an equilibrium state where their diffusion velocity cancels exactly. This occurs for a value of the abundance that we call the equilibrium abundance. As a consequence of the dependence of the various forces with depth the equilibrium abundances must also vary with depth. In this paper, $\mathrm{C}, \mathrm{N}, \mathrm{O}$ and $\mathrm{Si}$ equilibrium abundances achieved by diffusion in two series of white dwarf models will be discussed and compared to observations. The two series cover a range in effective temperature of 50 000-150 $000 \mathrm{~K}$ for He rich envelopes, and of $30000-100000 \mathrm{~K}$ for $\mathrm{H}$ rich envelopes. In one case the surface gravity is maintained constant $(\log g=8$.) while in the other case, the surface gravity is allowed to change with effective temperature, according to realistic evolutionary sequence for a $0,6 \mathrm{M} \odot$ white dwarfs (Koester and Schönberner 1986).

\section{Metals in hot non-DA white dwarfs}

In helium dominated envelopes, the low opacity is responsible for producing a high pressure in the atmosphere. This favors the radiative acceleration by increasing the collisional width of the absorption lines. On the other hand, the mass ratio of the diffusing particles in a helium dominated 
environment is less than the equivalent mass ratio in a hydrogen dominated environment. Both effects act in the same direction in reducing the downward diffusion velocity, or, alternatively, in producing larger equilibrium abundances, in helium atmospheres.

Typical equilibrium abundances of $\mathrm{C}, \mathrm{N}, \mathrm{O}$ and $\mathrm{Si}$ in a hot He envelope white dwarf are shown in figure 1. In this $T_{e}=100000 \mathrm{~K}, \log \mathrm{g}=8 \mathrm{model}$, carbon, nitrogen and oxygen are distributed with depth in two shells. The minima between the two shells correspond to the region in the star where the elements are in the noble gas state (CV, NVI, OVII). As is well known (Michaud et al. 1976; Vauclair, Vauclair and Greenstein 1979) resonance lines in the noble gas state are at much shorter wavelengths than the range where most of the continuum radiation flux is emitted. The resulting radiative acceleration experienced by the ions in this stage of ionization is too small to support the elements. The case of $\mathrm{Si}$ is somewhat more complicated due to the atomic structure. One generally finds an abundance distribution with two minima. The outermost minimum is due to the SiV noble gas.

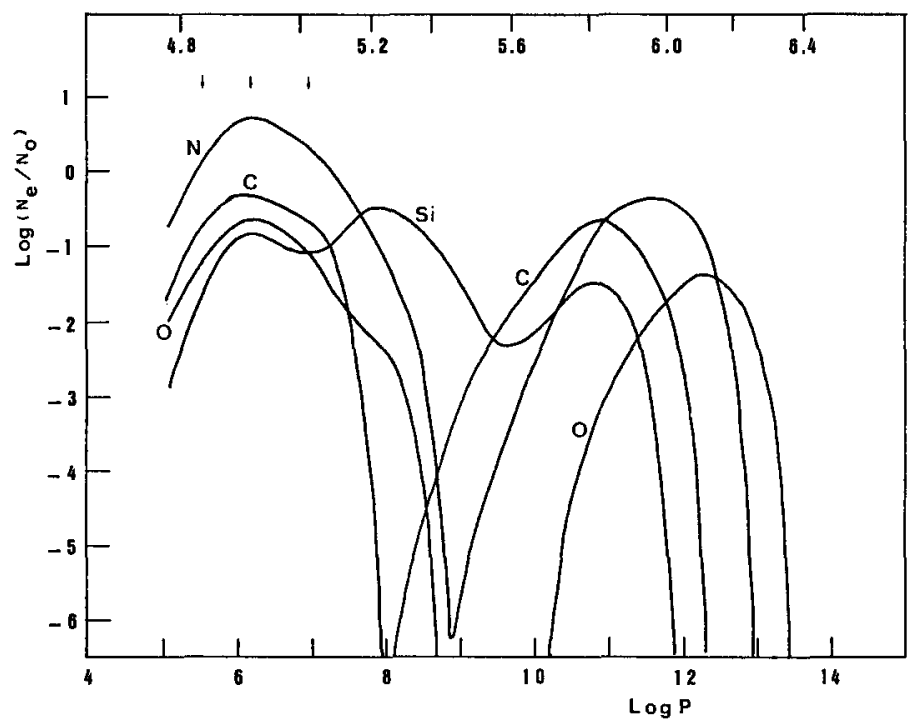

Figure 1. - C, N, O and Si diffusion equilibrium abundances in a $100000 \mathrm{~K}$, $\log \mathrm{g}=8.0 \mathrm{He}$ envelope. The abundances are given on logarithmic scale, in units of the solar abundance ratios. The lower scale is the gas pressure, while the upper scale is the temperature, both on logarithmic scale. The arrows show where the optical depth $\tau$ is $10^{-2}, 10^{-1}$ and 1 respectively. $\mathrm{C}, \mathrm{N}$ and $\mathrm{O}$ are distributed in two clouds with the minimum of the equilibrium abundance correcponding to the region where they are predominantly in the noble gas (CV, NVI, OVII. Si distribution reflects the more complex atomic structure and show three maxima. In this model, the maxima of the upper metallic clouds occur at $\tau \simeq 10^{-1}$, well inside the line forming region. 
How do the equilibrium atmospheric abundances vary with the effective temperature is illustrated in Figure 2. The Rosseland optical depth $\tau=0.1$ has been choosen as a representative value of the line forming region. Figure 2 shows the equilibrium abundances of $\mathrm{C}, \mathrm{N}, \mathrm{O}$ and Si predicted by diffusion at that particular optical depth. The abundance supported in the atmosphere depends heavily on the temperature: on one hand, increasing $T_{e}$ makes a larger radiation fux available for absorption; on the other hand, increasing $T_{e}$ favors a larger fraction of the noble gas ion into the line forming region. The interplay of these two effects is responsible for the behavior of the equilibrium abundances in Figure 2. Carbon is mainly CIV (at 70\%) at 50 $000 \mathrm{~K}$ with a small (2\%) fraction of $\mathrm{CV} . \mathrm{CV}$ increases to $20 \%$ at $60000 \mathrm{~K}$. This is not enough to balance the increased radiation flux. At higher temperature however, the increasing flux cannot balance the effect of the increasing fraction of $\mathrm{CV}(81 \%$ at $80000 \mathrm{~K}, 100 \%$ at $150000 \mathrm{~K})$ in reducing the radiative acceleration. This explains the decrease of the carbon equilibrium abundance with increasing temperature. The situation is similar for nitrogen and oxygen except that it takes higher an effective temperature to get the noble gas in noticeable amount at $\tau=0.1$. For silicon, the fraction of $\mathrm{SiV}$ in the $50000 \mathrm{~K}$ model is already of $75 \%$ and increases regularly up to $100000 \mathrm{~K}$ where it reaches almost $100 \%$. This is responsible for the dramatic decrease of the equilibrium abundance with increasing $T_{e}$. At temperature higher than $120000 \mathrm{~K}$, the fraction of $\mathrm{SiVI}$ starts to be large enough to make the radiative acceleration and the equilibrium abundance increasing with effective temperature. Negligeable at 50000 and $60000 \mathrm{~K}$, the effect on the equilibrium abundances of changing the surface gravity from the Hamada-Salpeter mass-radius relation to a more realistic evolutionary sequence (Koester and Schönberner 1986) cannot be neglected at higher effective temperature.

At this stage, how do the observations compare with the theory? At the very hot end of the white dwarf sequence, we even do not know the atmospheric chemical composition. The $\mathrm{H}$ and He poor object $\mathrm{H} 1504+65$ (Nousek et al. 1986) is presently the hottest known degenerate star at $T_{e} \simeq 160000 \mathrm{~K}$. While the absence of He lines at that temperature does not necessarily imply an helium poor atmosphere, ground based spectra definitely show OVI and CIV feature; OVI absorption at $1032 \AA$ was also seen in a Voyager spectrum. If we believe for the time being, i.e. until we are able to modelize $\mathrm{H} 1504+65$ atmosphere and derive relevant abundances, that this object could be a hot progenitor of the helium rich sequence, it is tempting to compare the predictions of diffusion theory with what is observed in that object. In the $150000 \mathrm{~K}$ model, carbon is almost entirely in the noble gas configuration, only a tiny fraction of $\simeq 10^{-3}$ still survives as $\mathrm{CIV}$. CV would not be detectable: its resonance lines are too far in the EUV and the only line to fall in the long wavelength range of IUE $(\lambda 2273 \AA)$ originates from the first excited level which has negligeable population. With a total $\mathrm{C} / \mathrm{He}=2.5-4.010^{-5}$, the fraction $\mathrm{CIV} / \mathrm{He}$ which could 

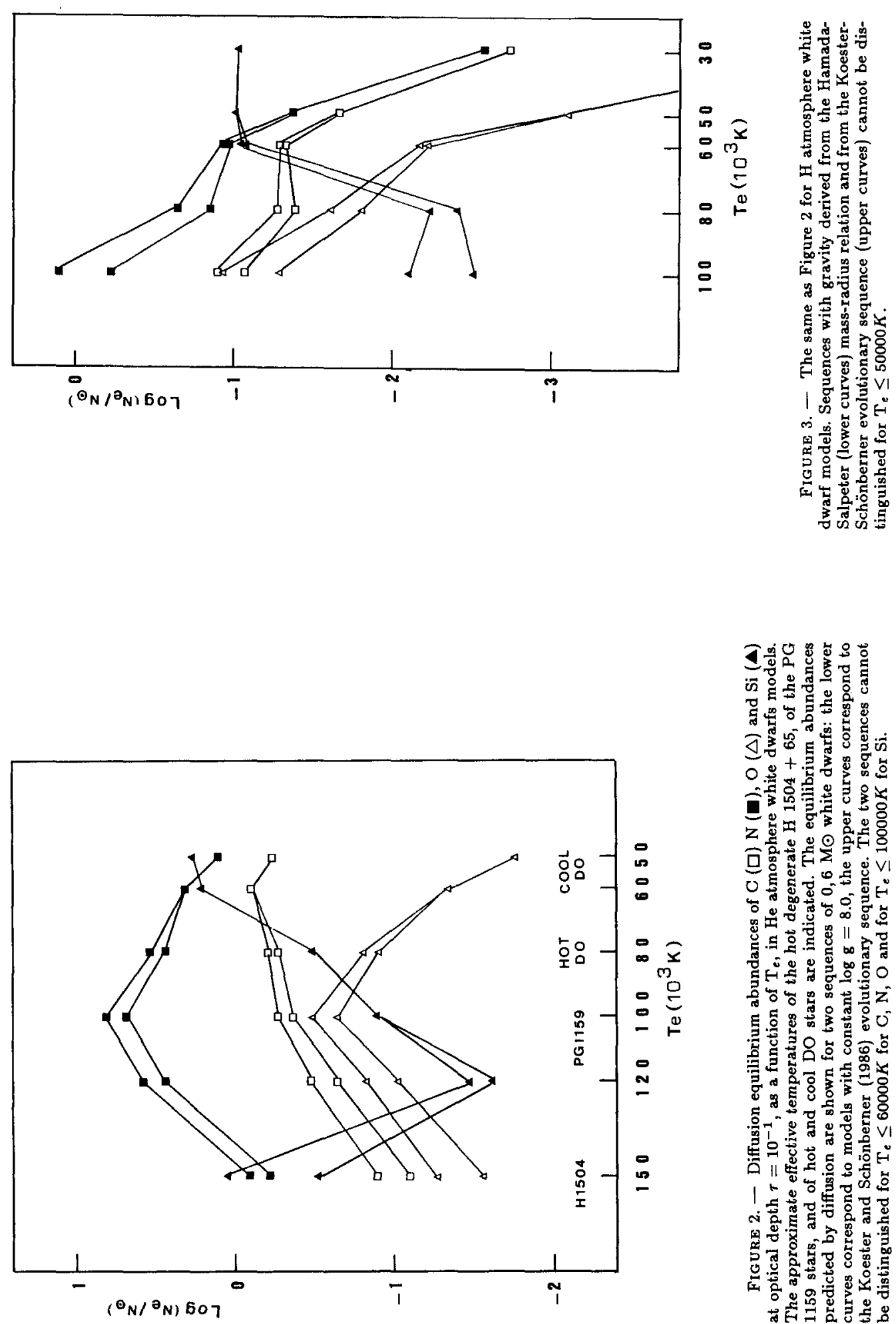
form the $\lambda 1550 \AA$ resonance line is $\simeq 2 .-5 . \times 10^{-8}$. Nitrogen is also mainly (at $98 \%$ ) in the noble gas stage of ionization. The resonance lines are far in the EUV. The IUE long wavelength range could not detect the 1901.5 $\AA$ line which originates from the first excited level, not populated at that temperature. $N V \lambda 1240 \AA$ should be present but only $2 \%$ of the total nitrogen is in the NV stage of ionization, mostly in excited states, a situation not favorable to the absorption from the ground state from which the line originate. Oxygen is approximately half OVI, half OVII $(46 \%$ and $53 \%$ respectively). This makes highly favorable the detection of $\mathrm{OVI}$ lines, as observed in $\mathrm{H}$ $1504+65$, especially through the line at $\lambda 1033.8 \AA$ which originates from the ground state. The OVI/He ratio in equilibrium at $\tau=0.1$ is $\simeq 8 \times 10^{-6}-3 \times 10^{-5}$. Silicon is mainly $\mathrm{Si} V$ and $\mathrm{Si} V I$ none of which does have line in the IUE domain. Silicon, if it is there with the high abundance predicted by theory (from 3 times less than solar to solar) would remain undetected.

The range of effective temperatures $100000 \mathrm{~K}-120000 \mathrm{~K}$ is the domain of the PG 1159 stars. Inspection of figure 2 reveals that in this temperature range the equilibrium abundances of $\mathrm{N}$ and $\mathrm{O}$ reach their maximum value. Is that a pure coincidence? At $100000 \mathrm{~K}$, carbon is still dominantly in the $\mathrm{CV}$ state but $\mathrm{CIV}$ accounts for almost $4 \%$. Carbon is depleted by only a factor 2 compared to the sun. $\mathrm{C} I V$ resonance lines should be strong at these temperatures, which is observed in many PG 1159 stars (Bond et al. 1984; Sion, Liebert and Starrfield 1985; Wesemael, Green and Liebert 1985) and in the related pulsating central star of the planetary nebula K1-16 (Grauer and Bond 1984). It is more difficult to understand why $N V \lambda 1240 \AA$ is not stronger in all stars of that temperature range. The predicted total nitrogen abundance at equilibrium is 5 to 7 times the solar value and $75 \%$ should be in the form of $\mathrm{NV}$. $\mathrm{NV}$ is detected in some PG 1159 stars (Bond et al. 84; Sion, Liebert and Starrfield 1985; Wesemael, Green and Liebert 1985) but it is generally a weak line. The star KPD $0005+5106$ also shows carbon lines but no nitrogen from low resolution IUE spectrum (Downes, Liebert and Margon 1985). Does this mean that nitrogen, which is always found more efficiently pushed upward by the radiative acceleration in our theoretical computations, has already been largely expulsed out of the star during the pre-PG 1159 phase, or depleted during previous nuclear burning episodes? Oxygen is only at $3 \%$ in the OVI stage but the dominant $O I V$ and $O V$ stages do not have strong lines in the IUE or visible wavelength range. With $\mathrm{O} / \mathrm{He} \simeq 1 \cdot-2 \cdot 10^{-4}$ (a deficiency of 6. -3 . compared to the Sun) the oxygen equilibrium abundance reaches its maximum at that temperature. As a consequence, oxygen absorption lines (OVI) are expected at this temperature. They are effectively observed in a number of PG 1159 stars (Sion, Liebert and Starrfield 1985). As far as silicon is concerned, it is hardly detectable being in the $\mathrm{Si} V$ ionization stage. A tiny fraction of surviving $\mathrm{Si} I V$ (a few $10^{-3}$ ) could be responsible for the weak SiIV barely seen in a few PG 1159 . We still do not 
have, at the time of writing, an abundance determination for PG 1159 stars. The present analysis, based on the assumption of an helium dominated envelope could become irrelevant to PG 1159 stars if the chemical composition is $\mathrm{C} / \mathrm{O}$ dominated, as suggested by Starrfield et al. (1984) to trigger the instability. I have suggested elsewhere (Vauclair 1987) that the diffusion mechanism which naturally produces equilibrium clouds of $\mathrm{C}, \mathrm{N}, \mathrm{O}$ and $\mathrm{Si}$ in the supposedly helium rich outer layers of these stars could be responsible for both the presence of absorption lines of these species in the stellar spectra and of the instability observed in some of the PG 1159 stars.

At lower effective temperature $(80000 \mathrm{~K})$ our computations may be relevant to the hot DO. For these stars, whose prototype is PG1034+001, one knowns that they are helium dominated. In our $80000 \mathrm{~K}$ models, carbon is still dominantly in the $\mathrm{CV}$ configuration but $\mathrm{CIV}$ reaches almost $20 \%$. Carbon is efficiently supported by the radiation field to approximately half the solar abundance $\left(\mathrm{C} / \mathrm{He} \simeq 2 \times 10^{-4}\right)$. This equilibrium value at $\tau=0.1$ is in good agreement with independent diffusion computation by Chayer et al. (1987) who find $\mathrm{C} / \mathrm{He} \simeq 4.10^{-4}$ at $\tau=1$. But it is significantly in disagreement with the value derived from the observation (Sion, Liebert and Wesemael 1985) which is smaller by a factor 100 . Nitrogen is found to be overabundant compared to the solar abundance (by a factor 3) which is in good agreement with the overabundance observed (Sion, Liebert and Wesemael 1985) and with the theoretical study of Chayer et al. (1987). Oxygen is found deficient by a factor 6-8 compared to the Sun, which is a factor 10 smaller than the value predicted at $\tau=1$ by Chayer et al. (1987). However the uncertainty on the observed abundance is large enough to accomodate the two independently derived theoretical values!

At the temperature of $60000 \mathrm{~K}-50000 \mathrm{~K}$, the lower limit we have considered here for hot non -DA, in order to avoid helium convection zones, we are in the domain of the cool DO as HD 149199B (Bruhweiler and Kondo 1983; Sion and Guinan 1983). Unfortunately we do not have abundance determinations to compare with theory for that star. CIV dominates at $\tau=0.1(74 \%$ at $60000 \mathrm{~K}, 70 \%$ at $50000 \mathrm{~K})$. Carbon is supported by the radiation flux at a value close to the solar abundance ( $80 \%$ of the solar abundance at $60000 \mathrm{~K}, 60 \%$ at $50000 \mathrm{~K}$ ). As a consequence strong $\mathrm{C} I V$ lines are expected and are observed. Nitrogen is predicted to be slightly overabundant compared to the Sun (by a factor 2 at $60000 \mathrm{~K}$ and 1.25 at $50000 \mathrm{~K}$ ). However it is in the form of $\mathrm{N} I I I$ and $\mathrm{N} I V$ which do not have strong lines in the visible or in the IUE range. Weaker NIII lines should be present but have not been detected. Oxygen is predicted to be deficient by larger factors (from 20 at $60000 \mathrm{~K}$ to 60 at $50000 \mathrm{~K}$ ). The dominant ionization stages are $O I I I$ and $\mathrm{O} I V$ which do not show lines neither in the visible nor in the UV accessible to IUE. While $\mathrm{SiV}$ is still dominant at this temperature, $\mathrm{Si} I V$ should be abundant enough $(6 \%$ at $60000 \mathrm{~K}$ and $24 \%$ at $50000 \mathrm{~K}$ ) to lead to absorption lines, especially at these temperatures where the theory predicts 
detected in HD 149499B (Bruhweiler and Kondo 1983; Sion and Guinan 1983).

\section{Metals in hot DA white dwarfs}

For hot hydrogen envelope white dwarfs we consider the effective temperature range $30000 \mathrm{~K}$ $-100000 \mathrm{~K}$, as there is no DA hotter than $100000 \mathrm{~K}$, or even hotter than $80000 \mathrm{~K}$ (Fleming, Liebert and Green 1986). The distribution of the equilibrium abundances are quite similar to the case discussed in figure1. The somewhat reduced radiative acceleration and enhanced downward acceleration make the amount of metals which may be supported smaller in hydrogen envelopes than in helium envelopes. Figure 3 shows the variations with effective temperature of the equilibrium abundances for $\mathrm{C}, \mathrm{N}, \mathrm{O}$ and $\mathrm{Si}$ computed at Rosseland optical depth $\tau=0.1$ in pure hydrogen atmospheres, with $\log g=8.0$ and along the $0.6 \mathrm{M} \odot$ evolutionary sequence of Koester and Schönberner (1986). A comparison with the similar figure 2 for helium envelopes illustrates the differences. In the considered temperature range, radiative levitation cannot support more than the solar abundances, in contrast with the helium case with the only exception of $\mathrm{N}$ at the very hot end of the Koester and Schönberner sequence.

In many hot DA, IUE observations have revealed the presence of metallic absorption. In some cases, velocity differences between metallic lines and Balmer lines point to the existence of a wind, or of circumstellar material. In such cases, the predictions of the pure diffusion may be irrelevant and mass loss should be introduced in some way as one additional physical mechanism competing with diffusion (Michaud 1987). We are faced then to the difficulty that we do not know how to introduce mass-loss in a physically consistent way. The diffusion computations which are presented here may apply to those cases where the observed metallic lines form in the stellar photosphere. It is the case in the well discussed Feige 24 star, in which resonance lines of $\mathrm{C} I V, \mathrm{~N} V$ and $\mathrm{Si} I V$ have been discovered in high resolution IUE spectra (Dupree and Raymond 1982). Diffusion equilibrium abundances have been computed for that star (Morvan, Vauclair and Vauclair 1986; Chayer et al. 1987). Nitrogen and silicon abundances predicted by the diffusion theory are in good agreement with the value deduced from the observations (Wesemael, Henry and Shipman 1984). Carbon is predicted too strong, as in the case of the hot DO PG 1034+001 discussed earlier. It is also the case in cooler DA stars as W 1346 (Bruhweiler and Kondo, 1983) or CD $-38^{\circ} 10980$ (Holberg et al., 1985) which do show only Si lines in their IUE spectra. Inspection of figure 3 shows that the radiatively supported abundances of $\mathrm{C}, \mathrm{N}, \mathrm{O}$ dramatically decrease with decreasing effective temperature. These elements are no more supported at the $\mathrm{W} 1346$ and $\mathrm{CD}-38^{\circ} 10980$ effective temperatures, while $\mathrm{Si}$ remains radiatively supported to approximately $1 / 10$ of the solar abundance. Diffusion predictions seem to be in qualitative agreement with the observations, but the Si abundance observed in W 1346, while uncertain by large a factor (Wesemael, Henry and a maximum for the $\mathrm{Si}$ abundance (at about twice the solar abundance). But $\mathrm{Si} I V$ has not been 


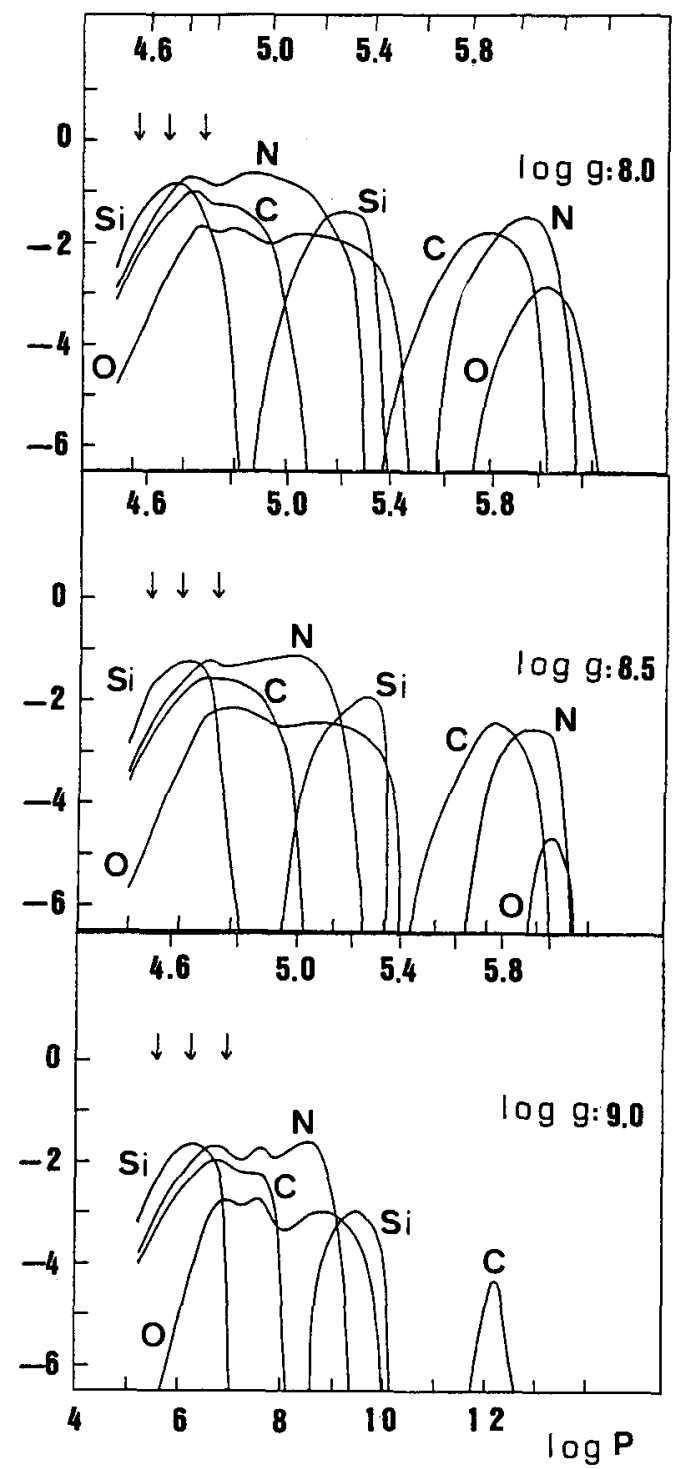

Figure 4. - Dependence of the metal clouds on changes in the surface gravity. The diffusion equilibrium abundances of $\mathrm{C}, \mathrm{N}, \mathrm{O}$ and $\mathrm{Si}$ in $\mathrm{H}$ white dwarf models supposed to represent $\mathrm{PG} 1210+533$ at $\mathrm{T}_{e}$ $=56000 \mathrm{~K}$, are shown for $\log \mathrm{g}=8.0,8.5,9.0$. Each panel is similar to figure 1, with the lower scale of the bottom panel showing the common pressure scale while the upper scale in each panel indicates the run of the temperature in individual model. While the decrease of the equilibrium abundances with increasing gravity may be clearly seen, the $\mathrm{C}, \mathrm{N}$ and $\mathrm{Si}$ abundances predicted in the line forming region are still large enough, even at $\log g=9.0$, to lead to absorption lines, which are not observed. 
Shipman 1984), in still less than the one predicted by the diffusion theory (Morvan, Vauclair and Vauclair, 1986; Chayer et al. 1987).

There are at least two puzzling questions related to the hot DA white dwarfs: i) at the same effective temperature, some DAs with metallic lines show evidence of ongoing mass-loss, some other do not; for instance at about 62 000K, G 191-B2B shows evidence of mass loss (Bruhweiler and Kondo 1981) while Feige 24 does not; ii) at the same effective temperature, some DAs do show metallic lines, some other do not: while Feige 24 shows metallic lines, HZ 43 and PG $1210+533$ at similar temperature do not. One may argue that the balance between the upward radiative acceleration and the downward acceleration due to pressure and thermal gradients strongly depends on the gravity (i.e. the mass) of the white dwarf. It has been found indeed that both HZ 43 (Holberg et al. 1986) and PG 1210+533 (Holberg et al. 1988) have higher than average $\log \mathrm{g}$. A test of the equilibrium abundances dependence on gravity as been performed in models of $\mathrm{PG} 1210+533$ ( $\mathrm{T}_{e} \simeq 56000 \mathrm{~K}$ ). Figure 4 shows $\mathrm{C}, \mathrm{N}, \mathrm{O}$ and $\mathrm{Si}$ clouds in equilibrium for three values of the surface gravity $\log g=8.0 ; 8.5 ; 9.0$. In spite of the decrease of the abundances with increasing gravity, there is still sufficient amount of $\mathrm{C}, \mathrm{N}$ and $\mathrm{Si}$ in the line forming region to produce lines which have not been observed in a high resolution IUE spectrum. The sensitivity of diffusion equilibrium on gravity does not seem to be large enough to explain the absence of metals in high gravity hot hydrogen white dwarfs like HZ 43 or PG $1210+533$. Consequently, interpreting the coexistence at the same effective temperature of DA stars with and without metals in their atmosphere remains a puzzle.

\section{Conclusion}

In the hot white dwarf stars, the radiative acceleration through absorption lines may be efficient enough to balance the gravitational field. An equilibrium state results where the elements are inhomogeneously distributed with depth. The theoretical computations have shown that $\mathrm{C}$, $\mathrm{N}, \mathrm{O}$ and $\mathrm{Si}$ may be supported in both hydrogen and helium rich white dwarf outer layers. A comparison with observations however is only qualitatively satisfactory at the present time. Some problems remain unsolved: i) nitrogen is observed to be weak in the PG 1159 stars while the theory predicts an overabundance of $\mathrm{N} / \mathrm{He}$ compared to the solar $\mathrm{N} / \mathrm{H}$ ratio, for helium rich envelopes in the relevant effective temperature range; ii) similarly, in the DO white dwarfs, carbon is predicted too strong compared to observations of hot $\mathrm{DO}$, and $\mathrm{Si}$, predicted also to be abundant in cool DO is not observed; iii) in hot hydrogen white dwarfs, we are faced to the unsolved problems of the coexistence, at the same temperature, of stars with ongoing mass fiows, and of stars without observable mass flow where the absorption lines form in the photosphere; and the coexistence at the same temperature of white dwarfs with and without metallic absorption lines. The suspected 
role of the surface gravity in solving this difference seems to have been overestimated.

In the theoretical computations summarized here, the assumption was implicitly made that there were enough diffusing particles in the stellar outer layers to fill in the cloud and reach the equilibrium abundance predicted by diffusion. However, one must keep in mind that one does not know the original metal abundances to start with the diffusion computation. What would happen if one of the considered species is missing or is present with such a low abundance that the corresponding equilibrium cloud could not be filled? One may expect that, because the equilibrium is not reached as a consequence of the abundance being too low the radiative acceleration should exceed the downward acceleration in that part of the atmosphere where the elements are not in the noble gas state. These elements could consequently be pushed out and leave the star in the form of a selective wind, as suggested by Vauclair, Vauclair and Greenstein (1979). In this case, the cloud predicted by the diffusion theory could not materialize.

The above mentionned difficulties point to the need for further theoretical progresses in the diffusion theory and in the stellar atmosphere modeling. Among other sources of uncertainty it should be outlined that the computations of the radiative acceleration at small optical depths should be improved, especially in the very hot white dwarfs where recent high dispersion IUE spectra reveal the importance of NLTE. Furthermore, if the observed elements are distributed inhomogeneously in the stellar outer layers, as predicted by diffusion, a number of other inconsistancies should be considered: i) the abundances derived from homogeneous model atmospheres cannot be directly compared to the diffusion predictions; synthetic spectra should include the inhomogeneous metal abundance distribution; ii) the flux distribution in model atmospheres itself may be affected by the presence of the metallic clouds; the differences with pure $\mathrm{H}$ or $\mathrm{He}$ atmosphere has to be evaluated. It should be larger in the atmospheres dominated by He, which has a low opacity compared to $\mathrm{H}$. Would metal clouds in the atmosphere solve the difficulty of fitting the energy distribution in $\mathrm{H} 1504+65$ with any existing model atmosphere? Last but not least, the question of the stability of such clouds should be addressed.

\section{References}

Bond, H. Grauer, A.D., Green, R.F. and Liebert, J. 1984, Astrophys. J. 279, 751

Bruhweiler, F.C. and Kondo, Y. 1981, Astrophys. J. Letters 248, L 123

BruhweILer, F.C. and Kondo, Y. 1983, Astrophys. J. 269, 657

Chayer, P., Fontaine, G., Wesemael, F. and Michaud, G. 1987 in IAU Colloquium $n^{\circ} 95$ :

The Second Conference on Faint Blue Stars, A.G.D. Philip, D.S. Hayes and J. Liebert, eds.,

L. Davis Press, Schenectady, p. 653

Downes, R.A., Liebert, J. and Margon, B. 1985, Astrophys. J. 290, 321

Dupree, A.K. and RAYmond, J.C. 1982, Astrophys. J. Letters 263, L63

Fleming, T., Liebert, J. and Green, R.F. 1986, Astrophys. J. 308, 176 
Fontaine, G. and Michaud, G. 1979, Astrophys. J. 231, 826

Grauer, A.D. and Bond, H.E. 1984, Astrophys. J. 277, 211

Holberg, J., Wesemael, F. and Basile, J. 1986, Astrophys. J. 306, 629

Holberg, J., Sion, E.M., Liebert, J. and VAuclair, G. 1988, in "A Decade of UV Astronomy with the IUE satellite", ESA SP-281, in press

Holberg, J., Wesemael, F., Wegner, G. and Bruhweiler, F.C. 1985, Astrophys. J. 293, 294

Koester, D. and Schönberner, D. 1986, Astron. Astrophys. 154, 125

Michaud, G. 1987, in IAU Colloquium $n^{\circ}$ 95; The Second Conference on Faint Blue Stars, A.G.D. Philip, D.S. Hayes and J. Liebert, eds., L. David Press, Schenectady, p. 249

Michaud, G., Charland, Y., Vauclair, S. and Vauclair, G. 1976, Astrophys. J. 210, 447

Morvan, E., Vauclair, G. and Vauclair, S. 1986, Astron. Astrophys. 163, 145

Nousek, J.A., Shipman, H.L., Holberg, J.B., Liebert, J., Pravdo, S.H., White, N.E. and Giommi, P. 1986, Astrophys. J. 309, 230

Schatzman, E. 1958, White dwarfs, North-Holland Publ. Comp., Amsterdam

ShIPMAN, H.L. 1987, in IAU Colloquium n ${ }^{\circ}$ 95: The Second Conference on Faint Blue Stars, A.G.D. Philip, D.S. Hayes and J. Liebert, eds., L. David Press, Schenectady, p. 273

Sion, E.M. and Guinan, E.F. 1983, Astrophys. J. Letters 265, L87

Sion, E.M., Liebert, J. and Starrfield, S.G. 1985, Astrophys. J. 292, 471

Sion, E.M., Liebert, J. and Wesemael, F. 1985, Astrophys. J. 292, 477

Starrfield, S.G., Cox, A., Kidman, R.B. and Pesnell, W.D. 1984, Astrophys. J. 281, 800

VAUCLAIR, G. 1987, in IAU Colloquium $\mathrm{n}^{\circ}$ 95: The Second Conference on Faint Blue Stars, A.G.D. Philip, D.S. Hayes and J. Liebert, eds., L. David Press, Schenectady, p. 341

VAUCLAIR, G. and Liebert, J. 1987 in Exploring the Universe with the IUE Satellite, Y. Kondo ed., Reidel, Dordrecht, p. 344

Vauclair, G., Vauclair, S. and Greenstein, J.L. 1979, Astron. Astrophys. 80, 79

VAuclair, S. 1983, in Astrophysical Processes in Upper Main Sequence Stars, 13th Advanced Course of the Swiss Society of Astronomy and Astrophysics, eds. B. Hauck and A. Maeder, Geneva Observatory

Wesemael, F., Green, R.F. and Liebert, J. 1985, Astrophys. J. Suppl. 58, 379

Wesemael, F., Henry, R.B.C. and Shipman, H.L. 1984, Astrophys. J. 287, 868 